VOL. $24(1981), 221-225$.

\title{
ON THE DEGREE OF APPROXIMATION BY SZÁSZ OPERATORS
}

\section{SuREsh Prasad Singh}

The aim of the present note is to give the degree of approximation by Szász operators.

\section{Introduction}

The linear positive operators $\left(S_{n} f\right)$ defined as

$$
\left(S_{n} f\right)(x)=\left(S_{n}(f(t) ; x)\right)=e^{-n x} \sum_{k=0}^{\infty}\left((n x)^{k} /\lfloor k) f(k / n)\right.
$$

were introduced by Szász [3] to approximate $f \in C[0, \infty)$. Stancu [2] has given the following result in uniform norm.

THEOREM 1. Let $f \in C^{l}[0, a], a>0$, and let $\omega\left(f^{\prime} ; \cdot\right)$ be its modulus of continuity. Then, for $n \in N$,

$$
\left\|S_{n} f-f\right\| \leq(a+\sqrt[V]{ } a) \cdot 1 / \sqrt{ } n \cdot \omega\left(f^{\prime} ; 1 / V_{n}\right) .
$$

- Recently we [4] have obtained the estimate

$$
\left\|S_{n} f-f\right\| \leq(\operatorname{Va}+(a / 2)) \cdot 1 / V_{n} \cdot \omega\left(f^{\prime} ; 1 / V n\right)
$$

which is sharper than the corresponding estimate (1.2).

The object of the present note is to extend the result (1.3) for

Received 25 March 1981. The author is thankful to Professor O.P. Varshney, University of Roorkee (India) for his valuable suggestions in this paper. The author would also like to thank the University Grants Commission for providing funds. 
$f \in c^{p+1}[0, a], r=0,1,2, \ldots$.

We prove the following.

THEOREM 2. Let $f \in c^{p+1}[0, a], a>0$, and let $\omega\left(f^{(p+1)}\right.$; $)$ be its modulus of continuity. Then, for $n \in N$,

(1.4) $\left\|S_{n}^{(r)} f-f^{(r)}\right\| \leq r / n \cdot\left\|f^{(r+1)}\right\|+K_{n, r} \cdot 1 / V_{n} \cdot \omega\left(f^{(r+1)} ; 1 / V_{n}\right)$

where

$$
K_{n, r}=\left[(a / 2)+\left(r / 2 V_{n}\right)+\left(r^{2} / 4 n\right)+\left(\left(r^{2} / 4 n\right)+a\right)^{\frac{3}{2}} \cdot\left(1+\left(r / 2 V_{n}\right)\right)\right]
$$

\section{Proof}

We use the following results [1],

$$
\begin{aligned}
\left(S_{n}\right)(x) & =1, \\
\left(S_{n}(t-x)\right)(x) & =0, \\
\left(S_{n}(t-x)^{2}\right)(x) & =x / n .
\end{aligned}
$$

After differentiating (1.1) $r$ times with respect to $x$, we get

$$
\text { (2.4) } \quad\left(S_{n}^{(r)} f\right)(x)=n^{r} e^{-n x} \sum_{k=0}^{\infty}\left((n x)^{k} /\lfloor k) \Delta_{n}^{r} \cdot f(k / n) \quad(r \leq n)\right. \text {, }
$$

where $\Delta_{n}^{r} f(k / n)$ represents the difference of order $r$ of the function $f$ with step $I / n$ starting from value $k / n$. This difference of order $r$ is defined by

$$
\Delta_{n^{-1}}^{l} f(k / n)=\Delta_{n^{-1}} f(k / n)=f((k+I) / n)-f(k / n)
$$

and

$$
\Delta_{n^{-1}}^{r+1} f(k / n)=\Delta n^{-1}\left(\begin{array}{c}
\Delta_{n}^{r} \\
-1
\end{array}(k / n)\right), \quad r=1,2, \ldots .
$$

By using the mean value theorem,

$$
\Delta_{n}^{r} f(k / n)=\left(1 / n^{r}\right) f^{(r)}\left(\left(k+r \theta_{k}\right) / n\right), \theta_{k} \in(0,1) .
$$


With the help of (2.4) and (2.5),

$$
\left(S_{n}^{(r)} f(x)\right)=e^{-n x} \sum_{k=0}^{\infty}\left((n x)^{k} /\lfloor k) f^{(r)}\left(\left(k+r \theta_{k}\right) / n\right)\right.
$$

We know that

$$
\text { (2.7) } \begin{aligned}
f^{(r)}(x)-f^{(r)}\left(\left(k+r \theta_{k}\right) / n\right) \\
\quad=\left(x-\left(\left(k+r \theta_{k}\right) / n\right)\right) f^{(r+1)}(x)+\int_{x}^{\left(k+r \theta_{k}\right) / n}\left[f^{(r+1)}(x)-f^{(r+1)}(n)\right] d n .
\end{aligned}
$$

Using (2.6), (2.7) and the inequality

$$
\left|f^{(r+1)}(x)-f^{(r+1)}(n)\right| \leq[1+(|\eta-x| / \delta)] \cdot \omega\left(f^{(r+1)} ; \delta\right) \quad(\delta>0),
$$

we obtain that

$$
\begin{aligned}
& \left|f^{(r)}(x)-\left(S_{n}^{(r)} f\right)(x)\right| \\
& \leq\left|f^{(r+1)}(x)\right| \cdot \mid e^{-n x} \sum_{k=0}^{\infty}\left((n x)^{k} /\lfloor k)\left(x-\left(\left(k+r \theta_{k}\right) / n\right)\right) \mid\right. \\
& \quad+\omega\left(f^{(r+1)} ; \delta\right) e^{-n x} \sum_{k=0}^{\infty}\left((n x)^{k} /\lfloor k)\left|\int_{x}^{\left(k+r \theta_{k}\right) / n}[1+(|n-x| / \delta)] d n\right|\right. \\
& =S_{1}+S_{2} \text { (say). }
\end{aligned}
$$

Clearly

$$
\begin{aligned}
S_{1} & =\left|f^{(r+1)}(x)\right| \cdot \mid e^{-n x} \sum_{k=0}^{\infty}\left((n x)^{k} /\lfloor k)\left[x-(k / n)-\left(r \theta_{k} / n\right)\right] \mid\right. \\
& \leq\left|f^{(r+1)}(x)\right| \cdot r / n \cdot e^{-n x} \sum_{k=0}^{\infty}\left((n x)^{k} /\lfloor k)\left|\theta_{k}\right|\right. \\
& \leq r / n \cdot\left\|f^{(r+1)}\right\|,
\end{aligned}
$$




$$
\begin{aligned}
S_{2}= & \omega\left(f^{(r+1)} ; \delta\right) e^{-n x} \sum_{k=0}^{\infty}\left((n x)^{k} /\lfloor k)\right. \\
\times & \times\left[\left|\left(\left(k+r \theta_{k}\right) / n\right)-x\right|+(1 / 2 \delta)\left(\left(\left(k+r \theta_{k}\right) / n\right)-x\right)^{2}\right] \\
\leq & \omega\left(f^{(r+1)} ; \delta\right) e^{-n x} \sum_{k=0}^{\infty}\left((n x)^{k} /\lfloor k)\right. \\
\times & {\left[|x-(k / n)-(r / 2 n)|+(r / 2 n)+(1 / 2 \delta)(|x-(k / n)-(r / 2 n)|+(r / 2 n))^{2}\right] } \\
\leq & \omega\left(f^{(r+1)} ; \delta\right)\left[\left((r / 2 n)+\left(r^{2} / 8 n^{2} \delta\right)\right)+(1 / 2 \delta) e^{-n x} \sum_{k=0}^{\infty}\left((n x)^{k} /\lfloor k)\right.\right. \\
& \times(x-(k / n)-(r / 2 n))^{2}+(1+(r / 2 n \delta)) e^{-n x} \sum_{k=0}^{\infty}\left((n x)^{k / \mid k)|x-(k / n)-(r / 2 n)|]} .\right.
\end{aligned}
$$

From (2.1), (2.2), (2.3) we know that

$$
e^{-n x} \sum_{k=0}^{\infty}\left((n x)^{k} /\lfloor)(x-(k / n)-(r / 2 n))^{2}=x / n+r^{2} / 4 n^{2}\right.
$$

and

$$
\begin{aligned}
& e^{-n x} \sum_{k=0}^{\infty}\left((n x)^{k} / \mid k\right)|x-(k / n)-(r / 2 n)| \\
& \leq \sqrt{e^{-n x} \sum_{k=0}^{\infty}\left((n x)^{k} /\lfloor k)(x-(k / n)-(r / 2 n))^{2} \cdot\left(s_{n} 1\right)(x)\right.} \\
&=\left(\left(r^{2} / 4 n^{2}\right)+(x / n)\right)^{\frac{3}{2}} .
\end{aligned}
$$

By choosing $\delta=1 / \sqrt{n}$, we finally get, for all $x \in[0, a]$, that

$$
S_{2} \leq K_{n, r} \cdot 1 / \sqrt{n} \cdot \omega\left(f^{(r+1)} ; 1 / \sqrt{n}\right) .
$$

This completes the proof.

COROLLARY 1. If $f^{(r+1)} \in \operatorname{Lip}_{M} \alpha, 0<\alpha \leq 1, M>0$, then, for $n \in N$,

$$
\left\|S_{n}^{(r)} f-f^{(r)}\right\| \leq r / n \cdot\left\|f^{(r+1)}\right\|+M \cdot K_{n, r} \cdot n^{-(\alpha+1) / 2} .
$$

COROLLARY 2. If, in addition to the hypotheses of Theorem 2, $f \in c^{r+2}[0, a]$, then, for $n \in N$, 


$$
\left\|S_{n}^{(r)} f-f^{(r)}\right\| \leq r / n \cdot\left\|f^{(r+1)}\right\|+K_{n, r^{l n}} \cdot\left\|f^{(r+2)}\right\| .
$$

\section{References}

[1] T. Hermann, "On the Szász-Mirkian operator", Acta Math. Acad. Sci. Hungar. 32 (1978), 163-173.

[2] D.D. Stancu,"Use of probabilistic methods in the theory of uniform approximation of continuous functions", Rev. Roumaine Math. Pures App 2. 14 (1969), 673-691.

[3] Otto Szasz, "Generalization of S. Bernstein's polynomials to the infinite interval", J. Res. Nat. Bur. Standards 45 (1950), $239-245$.

[4] Om P. Varshney and Suresh P. Singh, "On degree of approximation by positive linear operators", Rend. Mat. (to appear).

Department of Mathematics,

University of Roorkee,

Roorkee (U.P.) 247672,

India. 